\title{
Major Sources of Plant Fodder Species of South East Nigeria, Associated Livestock, Percentage Preferences, a Few Physiognomic Attributes and Potential New Sources
}

\author{
Ukpaka, Chukwujekwu G.
}

Department of Biological Sciences, Faculty of Natural Sciences, Chukwuemeka Odumegwu Ojukwu University, Anambra State Nigeria

Author's e-mail: nellaevans20@gmail.com

\begin{abstract}
Sixty (60) species of plant fodder belonging to 34 families were utilized in this study. Goat, sheep and rabbits were the experimental livestock. For a period of one year, each species was offered to the animals first thing in the morning. Attitude (reaction) of each animal to each plant species were observed and recorded. Observations revealed that most species were acceptable on a daily bases. Most species were completely consumed ravenously: (Persea, Gmelina, Zea, Ficus, Ricinodendron, Vernonia, Colocasia, Elacis). Others were consumed more reluctantly but consumed nevertheless (Cola, Cocos, Treculia, Mangitena, Psidicem, Banbusa);Insome cases, the fruits were preferred to the leaves (Citrus, Carica, Treculia, Dacryodes, Dioscorea, Mangifera, Arachis, Abelmoscus, Musa). In others, leaves were preferred to fruits (Dialum, Milicia, Pterocarpus, Elaeis, Capsicum, Telfairia). The goats fed more voraciously than others. The rabbits preferred tender and softer food (Daucus, Tridox, Setaria, Bambusa, Ipomoea, Andropogon, Vernonia, Zea (et cetera). Most species were evergreen perennials, therefore food was avaible all year round. Potential new sources of fodder were also suggested (Ceiba, Spondias, Vitex, Justicia, Duranta, Mariscus). Waste products of harvest and discarded peels and rinds of foodstuff were also taken into consideration.
\end{abstract}

Original Research Article

Received: $21^{\text {st }}$ Feb., 2018 Accepted: $11^{\text {th }}$ Mar., 2018 Published: $14^{\text {th }}$ Mar., 2018

Keywords:

Sources

Fodder

South East

Livestock

Physiognomy

\section{INTRODUCTION}

$\mathrm{M}$ ore than two-thirds of the estimated World population of goats (391 million) are found in the tropics and sub-tropics (FAO, 1973). The most important Centres for good-keeping are in Africa where in certain countries (Niger, Mauritania and Somalia) more than one goat is kept per person (FAO, 1973). There are 224 million goats in Nigeria and this is exceeded only by India with a goat population of 69.2 million (FAO, 1973). Moreover, the FAO (1966) found that goats provided over $25 \%$ of total lean meat consumed in Nigeria between 1963 and 1964. Many forest tree species provide fodder for livestock production, especially during the dry season when livestock feed becomes scarce and is of poor nutritional quality. Thus, availability of tree fodder enhances the productivity of livestock as well as of wild game. Since these animals contribute to human food supply, forest tree species contribute indirectly through them to human nutrition. Apart from the destruction of farms by livestock, herdsmen lop branches of trees conserved on farmland to provide fodder for their livestock. As fodder resources dwindle, wild game migrate to other areas where they can have access to sources of food supply. Consequently deforestation results in shortage of fodder supply and is detrimental to sustainable livestock and wildlife management (Otegbeye and Onyeanusi, 2006). The grazing pressure has been increasing with the increase in human and livestock populations. Consequently, uncontrolled heavy grazing is causing great damage to soil and vegetation due to compaction and trampling. This create gaps in the forest and retrogression in certain localities. Local people use trees, shrubs, and herbs as fodder for their livestock. Primarily, the members of the family Poaceae are used as fodder in both fresh and dry forms throughout the year (Abbasi et al., 2012).

In modern times with the harsh economic realities globally, global inflation, glut in crude oil prices; for countries like 
Nigeria, Agriculture is regaining prominence and sources of livestock food that will neither compete with nor drain man's already overstretched food sources is a very welcome idea. Even plant sources hitherto labelled unwanted, together with Agricultural /food wastes (plant leaves, stalks, glumes, fruit pericarp, rinds, tuber peelings, herbaceous stems, food fibre, end products of plant food processing) have become very important and in much demand these days. This justifies continuous research in this area. As established plant food sources are documented, potential sources of likely new sources of plant fodder will be exploited, to accumulate a broad based inexhaustible, renewable livestock food base that will ensure long term sustainability in this respect.

\section{MATERIALS AND METHODS}

This Research work was carried out in Nnokwa, Idemili South Local Government Area of Anambra State. Firstly a long shed was built for the animals (a fundamental requirement for shelter, if they most thrive). Several goats (billy, nanny and maturing kids); several sheep (local ram, sheep and young); and many rabbits (all white, all black and mixed colouration). They were positioned separately, with the rabbits in their cages (males and females). Each morning, they were given a different fodder species and their feeding observed. These observations were recorded. The ones they savoured were retained while the ones they disliked were removed immediately. Water was served twice daily. The shed and cages were swept out, first thing in the morning and last thing in the night. Ash and used motor oil were sprinkled round the shed to prevent ant infestation. It took a period of one year to observe their preference for particular fodder species. The fodder were tied to stakes for easy accessibility/consumption by the goats and sheep. Baskets were also used. That of the rabbits were placed inside the cages. Bits of human food and leftover food were also given at intervals. Pregnant ones were sheltered separately and mature offsprings were sold in the market. Other materials used included: Machetes, stakes, generator, torch light, rafters and calabashes, disinfectants, brooms, bowls, pans et cetera (Akobundu and Agyakwa, 1998; Nyananyo, 2006).

\section{RESULT AND DISCUSSION}

Results obtained from the study was presented in Table 1, 2 and 3.

Table 1: Major sources of plant fodder species of South East Nigeria and Associated Livestock.

\begin{tabular}{|c|c|c|c|c|c|c|c|c|}
\hline $\mathbf{S} / \mathbf{N}$ & $\begin{array}{l}\text { LOCAL } \\
\text { NAME }\end{array}$ & $\begin{array}{l}\text { COMMON } \\
\text { NAME }\end{array}$ & $\begin{array}{l}\text { SCIENTIFIC } \\
\text { NAME }\end{array}$ & $\begin{array}{l}\text { PART } \\
\text { EATEN }\end{array}$ & GOAT & $\begin{array}{l}\text { CONSUMER } \\
\text { SHEEP }\end{array}$ & RABBIT & OTHERS \\
\hline 1 & Ogbu (tree) & & Ficus elasticoides & leaf & $\sqrt{ }$ & $\sqrt{ }$ & & \\
\hline 2 & Okwe & Nut tree & $\begin{array}{l}\text { Ricinodendron } \\
\text { heudeloti }\end{array}$ & $\sqrt{ }$ & $\sqrt{ }$ & $\sqrt{ }$ & & \\
\hline 3 & Nkwu & Oil palm & Elaeis guineensis & $\begin{array}{l}\text { Leaf pericarp, } \\
\text { kernel }\end{array}$ & $\sqrt{ }$ & $\sqrt{ }$ & & \\
\hline 4 & Ube oyibo & $\begin{array}{l}\text { English } \\
\text { pear }\end{array}$ & Persea americana & $\begin{array}{l}\text { Leaf, seed, } \\
\text { pericarp (fruit) }\end{array}$ & $\sqrt{ }$ & $\sqrt{ }$ & $\sqrt{ }$ & \\
\hline 5 & Ube Igbo & Local pear & Dacryodes edulis & Leaf, fruit, seed & $\sqrt{ }$ & $\sqrt{ }$ & & \\
\hline 6 & Ji (Stem tuber) & Yam & Dioscoreae species & $\begin{array}{l}\text { Tuber peel } \\
\text { (rind) }\end{array}$ & $\sqrt{ }$ & $\sqrt{ }$ & & \\
\hline 7 & Ede (root tuber) & Cocoyam & $\begin{array}{l}\text { Colocasia } \\
\text { esculenta }\end{array}$ & $\begin{array}{l}\text { Tuber, peel } \\
\text { (rind) }\end{array}$ & $\sqrt{ }$ & $\sqrt{ }$ & & \\
\hline 8 & $\begin{array}{l}\text { Unere (Giant } \\
\text { forb) }\end{array}$ & Banana & Musa sapientum & Leaf, fruit, peel & $\sqrt{ }$ & $\sqrt{ }$ & & Fowl \\
\hline 9 & $\begin{array}{l}\text { Ogede/ojoko } \\
\text { (giant forb) }\end{array}$ & Plantain & Musa paradisiaca & Leaf, fruit, peel & $\sqrt{ }$ & $\sqrt{ }$ & $\sqrt{ }$ & Fowl \\
\hline 10 & Oka & Maize & Zea mays & Entire plant & $\sqrt{ }$ & $\sqrt{ }$ & & \\
\hline 11 & Mangoro & Mango & Mangifera indica & Leaf, fruit & $\sqrt{ }$ & $\sqrt{ }$ & & \\
\hline 12 & Gova & Guava & Psidium guajava & Leaf, fruit, seed & $\sqrt{ }$ & $\sqrt{ }$ & & \\
\hline 13 & Akpu (shrub) & Cassava & Manihot esaulentus & $\begin{array}{l}\text { Leaf (preferable } \\
\text { dried) tuber }\end{array}$ & $\sqrt{ }$ & $\sqrt{ }$ & & \\
\hline 14 & Ugiri & Bushmango & Irvingia gabonensis & Leaf, fruit & $\sqrt{ }$ & $\sqrt{ }$ & & \\
\hline 15 & & Gmelina & Gmelina arborea & Leaf & $\sqrt{ }$ & $\sqrt{ }$ & & \\
\hline 16 & Icheku & $\begin{array}{l}\text { Velvet } \\
\text { tamarind }\end{array}$ & Dialum guinesense & Leaf fruit pulp & $\sqrt{ }$ & $\sqrt{ }$ & & \\
\hline 17 & Orji & Iroko & Milicia excelsa & leaf & $\sqrt{ }$ & $\sqrt{ }$ & & \\
\hline 18 & Oji & Kola & $\begin{array}{l}\text { Cola nitida, cola } \\
\text { acuminata }\end{array}$ & $\begin{array}{l}\text { Leaf seed } \\
\text { (preferably } \\
\text { dried) }\end{array}$ & $\sqrt{ }$ & $\sqrt{ }$ & & \\
\hline 19 & Akuoyibo & Coconut & Cocos nucifera & Leaf, kernel & $\sqrt{ }$ & $\sqrt{ }$ & & \\
\hline 20 & Oha & & $\begin{array}{l}\text { Pterocarpus } \\
\text { soyauxii }\end{array}$ & leaf & $\sqrt{ }$ & $\sqrt{ }$ & & \\
\hline 21 & Udara & & $\begin{array}{l}\text { Chrysophyllum } \\
\text { albidum }\end{array}$ & Leaf, fruit & $\sqrt{ }$ & $\sqrt{ }$ & & \\
\hline 22 & Ukwa & $\begin{array}{l}\text { African } \\
\text { breadfruit }\end{array}$ & Treculia africana & Leaf, fruit & $\sqrt{ }$ & $\sqrt{ }$ & & \\
\hline 23 & Uturu & & Nauclea diderichi & Leaf fruit & $\sqrt{ }$ & $\sqrt{ }$ & & \\
\hline
\end{tabular}


Table 1: (Continued)

\begin{tabular}{|c|c|c|c|c|c|c|c|c|}
\hline $\mathbf{S} / \mathbf{N}$ & $\begin{array}{l}\text { LOCAL } \\
\text { NAME }\end{array}$ & $\begin{array}{l}\text { COMMON } \\
\text { NAME }\end{array}$ & $\begin{array}{l}\text { SCIENTIFIC } \\
\text { NAME }\end{array}$ & $\begin{array}{l}\text { PART } \\
\text { EATEN }\end{array}$ & GOAT & $\begin{array}{l}\text { CONSUMER } \\
\text { SHEEP }\end{array}$ & RABBIT & OTHERS \\
\hline 24 & Ahaba & & $\begin{array}{l}\text { Dactyledenia } \\
\text { barteri }\end{array}$ & leaf & $\sqrt{ }$ & $\sqrt{ }$ & & \\
\hline 25 & Okwurubekee & pawpaw & Carica papaya & Leaf fruit seed & $\sqrt{ }$ & $\sqrt{ }$ & & Snail fowl \\
\hline 26 & & Soursop & Annona muricata & Fruit & $\sqrt{ }$ & $\sqrt{ }$ & & \\
\hline 27 & Oroma & Orange & Citrus species & Leaf, fruit, seed & $\sqrt{ }$ & $\sqrt{ }$ & & \\
\hline 28 & Achara (shrub) & Bamboo & Bambusa vulgaris & Leaf & $\sqrt{ }$ & $\sqrt{ }$ & & \\
\hline 29 & & $\begin{array}{l}\text { Elephant } \\
\text { Grass }\end{array}$ & $\begin{array}{l}\text { Pennisetum } \\
\text { purpureum }\end{array}$ & Leaf & $\sqrt{ }$ & $\sqrt{ }$ & & \\
\hline 30 & & $\begin{array}{l}\text { Gamba } \\
\text { grass }\end{array}$ & $\begin{array}{l}\text { Andropogon } \\
\text { tectorum }\end{array}$ & Leaf & $\sqrt{ }$ & $\sqrt{ }$ & & \\
\hline 31 & & $\begin{array}{l}\text { Guifffnea } \\
\text { grass }\end{array}$ & Panicum maximum & Leaf & $\sqrt{ }$ & $\sqrt{ }$ & & \\
\hline 32 & & Rice & Oryza sativa & Entire plant & $\sqrt{ }$ & $\sqrt{ }$ & & \\
\hline 33 & & & Eleusine indica & Leaf & $\sqrt{ }$ & $\sqrt{ }$ & & \\
\hline 34 & & & Setaria barbata & leaf & $\sqrt{ }$ & $\sqrt{ }$ & $\sqrt{ }$ & fowl \\
\hline 35 & & & $\begin{array}{l}\text { Dactyloctenium } \\
\text { aegyptium }\end{array}$ & $\begin{array}{l}\text { Leaf } \\
\text { inflorescence }\end{array}$ & $\sqrt{ }$ & $\sqrt{ }$ & & \\
\hline 36 & & $\begin{array}{l}\text { Bahama } \\
\text { grass }\end{array}$ & Cynodon dactylon & leaf & $\sqrt{ }$ & $\sqrt{ }$ & & \\
\hline 37 & & Ginger lily & Costusafer & Leaf & $\sqrt{ }$ & $\sqrt{ }$ & & \\
\hline 38 & & $\begin{array}{l}\text { Carrot } \\
\text { (stem tuber) }\end{array}$ & Daucuscarota & $\begin{array}{l}\text { Fruit } \& \\
\text { accessories }\end{array}$ & $\sqrt{ }$ & $\sqrt{ }$ & $\sqrt{ }$ & \\
\hline 39 & Akidi & Climber & Phaseolus vulgaris & & $\sqrt{ }$ & $\sqrt{ }$ & & \\
\hline 40 & Viovio shrub & & Cajanus cajans & & $\sqrt{ }$ & $\sqrt{ }$ & & \\
\hline 41 & Anara shrub & Eggplant & $\begin{array}{l}\text { Solanum } \\
\text { melangena }\end{array}$ & Leaf, fruit & $\sqrt{ }$ & $\sqrt{ }$ & & \\
\hline 42 & Ose shrub & Pepper & Capsicum anпиит & Leaf, fruit & $\sqrt{ }$ & $\sqrt{ }$ & & \\
\hline 43 & Onugbu shrub & & $\begin{array}{l}\text { Vernonia } \\
\text { amygdalina }\end{array}$ & leaf & $\sqrt{ }$ & $\sqrt{ }$ & & \\
\hline 44 & Shrub & & Ixora coccinea & leaf & $\sqrt{ }$ & $\sqrt{ }$ & & \\
\hline 45 & Shrub & & Aspilia africana & leaf & $\sqrt{ }$ & $\sqrt{ }$ & $\sqrt{ }$ & \\
\hline 46 & Shrub & Groundnut & Arachis hypogea & $\begin{array}{l}\text { Entire lea, fruit } \\
\text { plant }\end{array}$ & $\sqrt{ }$ & $\sqrt{ }$ & & \\
\hline 47 & Shrub & Pineapple & Ananas comosus & fruit & $\sqrt{ }$ & $\sqrt{ }$ & & \\
\hline 48 & Inine(forb) & & Amaranthus species & Leaf stem & $\sqrt{ }$ & $\sqrt{ }$ & & \\
\hline 49 & Ahihia(forb) & & Cochorus olitorius & Leaf stem & $\sqrt{ }$ & $\sqrt{ }$ & & \\
\hline 50 & Forb & & Tridax procumbens & Leaf & $\sqrt{ }$ & $\sqrt{ }$ & $\sqrt{ }$ & Fowl \\
\hline 51 & $\begin{array}{l}\text { Egwusi shrub } \\
\text { melon }\end{array}$ & & $\begin{array}{l}\text { Cucumeropsis } \\
\text { manii }\end{array}$ & Whole fruit & $\sqrt{ }$ & $\sqrt{ }$ & & \\
\hline 52 & Ugu climber & & $\begin{array}{l}\text { Telfairia } \\
\text { occidentalis }\end{array}$ & Lea, seed & $\sqrt{ }$ & $\sqrt{ }$ & & \\
\hline 53 & Shrub & & $\begin{array}{l}\text { Hibiscus } \\
\text { rosasinensis }\end{array}$ & Leaf & $\sqrt{ }$ & $\sqrt{ }$ & & \\
\hline 54 & Runner & & Ipomoea species & Leaf, tuber & $\sqrt{ }$ & $\sqrt{ }$ & $\sqrt{ }$ & \\
\hline 55 & Forn & & $\begin{array}{l}\text { Alternanthera } \\
\text { species }\end{array}$ & Leaf, stem & $\sqrt{ }$ & $\sqrt{ }$ & $\sqrt{ }$ & \\
\hline 56 & Climber & & $\begin{array}{l}\text { Desmodium } \\
\text { scorpiurus }\end{array}$ & Leaf stem & $\sqrt{ }$ & $\sqrt{ }$ & $\sqrt{ }$ & \\
\hline 57 & Shrub & Beans & Vigna unguiculata & Entire plant & $\sqrt{ }$ & $\sqrt{ }$ & & \\
\hline 58 & Shrub & Broomweed & Sida species & Leaf & $\sqrt{ }$ & $\sqrt{ }$ & & \\
\hline 59 & & Water leaf & $\begin{array}{l}\text { Talinum } \\
\text { triangulare }\end{array}$ & Entire plant & $\sqrt{ }$ & $\sqrt{ }$ & $\sqrt{ }$ & \\
\hline 60 & & Okro & $\begin{array}{l}\text { Abelmoscus } \\
\text { esculentus }\end{array}$ & Fruit & $\sqrt{ }$ & $\sqrt{ }$ & & \\
\hline 61 & Sugar cane & Cane sugar & $\begin{array}{l}\text { Saccharum } \\
\text { officinarum }\end{array}$ & Leaf, stem & $\sqrt{ }$ & $\sqrt{ }$ & $\sqrt{ }$ & \\
\hline 62 & Ukpodu & & $\begin{array}{l}\text { Napoleana } \\
\text { imperialis }\end{array}$ & Leaf, stem & $\sqrt{ }$ & $\sqrt{ }$ & & \\
\hline 63 & ububo & & $\begin{array}{l}\text { Alchornea } \\
\text { cordifolia }\end{array}$ & Leaf, stem & $\sqrt{ }$ & $\sqrt{ }$ & & \\
\hline 64 & & & Terminalia catappa & Fruit tree & & & & \\
\hline
\end{tabular}

From Table 1 above, one very significant feature of all the plant fodder species highlighted is that more than half of the total number also represent sources of human food delicacies.
It is also pertinent to note that while most of these plants are consumed raw preferably, a few are more palatable to the animals when sundried (Colocasia, Manihot et cetera). It is 
not advisable to give the leaves of Manihot and Cola species when fresh to the pregnant females. Rabbits being very fragile animals prefer the tenderest plant parts and it is important to note that what these animals eat is not restricted only to the ones highlighted, again animals from different localities may show varying degrees of preferences for each plant mentioned above.

Table 2: Family, Habit, Growth Form, Life Span and Percentage Preference of Fodder to Livestock.

\begin{tabular}{|c|c|c|c|c|c|c|}
\hline $\mathbf{S} / \mathbf{N}$ & SCIENTIFIC NAME & FAMILY & $\begin{array}{l}\text { GROWTH } \\
\text { FORM }\end{array}$ & LIFE SPAN & $\begin{array}{l}\text { OTHER ECONOMIC } \\
\text { VALUE }\end{array}$ & $\begin{array}{l}\text { PERCENTAGE } \\
\text { PREFERENCE } \\
\text { BY LIVESTOCK }\end{array}$ \\
\hline 1 & Ficus elasticoides & Moraceae & Tree & Perennial $\mathrm{H}$ & Domestic & A \\
\hline 2 & $\begin{array}{l}\text { Ricinodendron } \\
\text { heudeloti }\end{array}$ & Euphorbiaceae & Tree & Perennial W & Domestic & A \\
\hline 3 & Elaeis guineensis & Arecaceae & Tree & Perennial W & $\begin{array}{l}\text { Food, cash, industrial } \\
\text { domestic }\end{array}$ & A \\
\hline 4 & Persea americana & Lauraceae & Tree & Perennial W & Food, cash domestic & A \\
\hline 5 & Dacryodes edulis & Burseraceae & Tree & Perennial W & Food, cash, industrial & A \\
\hline 6 & Dioscoreasppe.galata & Dioscoreoceae & Stem tuber & Annual SW & Food, cash, industrial & A \\
\hline 7 & Colocasia esculenta & Araceae & Corm & Annual H & Food, cash, industrial & A \\
\hline 8 & Musa sapientum & Musaceae & Giant forb & Biennial H & Food, cash, industrial & A \\
\hline 9 & Musa paradisiaca & Musaceae & Giant forb & Biennial H & Food, cash, industrial & A \\
\hline 10 & Zea mays & Poaceae & Grass & Annual $\mathrm{H}$ & Food, cash, industrial & \\
\hline 11 & Mangifera indica & Anacardiaceae & Tree & Perennial W & Food, cash, industrial & A \\
\hline 12 & Psidium guajava & Myrtaceae & Tree & Perennial W & Food, cash, industrial & $\mathrm{B}$ \\
\hline 13 & Manihot esculentus & Euphorbiaceae & Root tuber & Perennial W & Food, cash, industrial & A \\
\hline 14 & Invingiagabonensis & Ixonanthaceae & Tree & Perennial W & Food, cash, domestic & A \\
\hline 15 & Gmelina arborea & Verbenaceae & Tree & Perennial W & Timber (3) domestic & A \\
\hline 16 & Dialum guineense & Caesalpiniaceae & Tree & Perennial W & $\begin{array}{l}\text { Food, cash, industrial } \\
\text { domestic }\end{array}$ & A \\
\hline 17 & Milicia excelsa & Moraceae & Tree & Perennial W & Timber (5) domestic & A \\
\hline 18 & Cola species & Sterculiaceae & Tree & Perennial W & Cash, domestic & $\mathrm{B}$ \\
\hline 19 & Cocos nucifera & Arecaceae & Tree & Perennial W & Food, cash, domestic & B \\
\hline 20 & Pterocarpus soyaxica & Fabaceae & Tree & Perennial W & Food, cash, domestic & B \\
\hline 21 & Chrysophylum albidum & Sapotaceae & Tree & Perennial W & Food, cash, domestic & B \\
\hline 22 & Treculia africana & Moraceae & Tree & Perennial W & $\begin{array}{l}\text { Food, cash, industrial } \\
\text { domestic }\end{array}$ & B \\
\hline 23 & Nauclea diderichii & Rubiacaceae & Tree & Perennial W & Timber (5) Food, domestic & B \\
\hline 24 & Dactyledema barteri & Sterculiaceae & Tree & Perennial W & Domestic & B \\
\hline 25 & Carica papaya & Caricaceae & Tree & $\begin{array}{l}\text { Perennial } \\
\text { SW }\end{array}$ & Food, cash, domestic & A \\
\hline 26 & Citrus species & Rutaceae & Tree & Perennial W & $\begin{array}{l}\text { Food, cash, industrial } \\
\text { domestic }\end{array}$ & B \\
\hline 27 & Annona murichata & Annonaceae & Tree & Perennial W & Food, cash & B \\
\hline 28 & Bambusa vulgaris & Poaceae & Shrub & Perennial W & Cash, industrial, Domestic & A \\
\hline 29 & Pennisetum purpurem & Poaceae & Grass & Perennial $\mathrm{H}$ & Domestic & B \\
\hline 30 & Andropogon tectorum & Poaceae & Grass & Perennial $\mathrm{H}$ & Domestic & A \\
\hline 31 & Panicum maximum & Poaceae & Grass & Perennial $\mathrm{H}$ & Domestic & $\mathrm{B}$ \\
\hline 32 & Oryza sativa & Poaceae & Grass & Annual H & Domestic & A \\
\hline 33 & Eleusine indica & Poaceae & Grass & Annual $\mathrm{H}$ & $\begin{array}{l}\text { Food, cash industrial(5) } \\
\text { domestic }\end{array}$ & A \\
\hline 34 & Setaria barbata & Poaceae & Grass & Annual H & Domestic (2) & A \\
\hline 35 & Cynodon dactylon & Poaceae & Grass & Annual H & Domestic (2) & B \\
\hline 36 & Costus afer & Poaceae & Grass & Annual $\mathrm{H}$ & Domestic (2) & B \\
\hline 37 & Daucus carota & Apiaceae & & Biannual $\mathrm{H}$ & $\begin{array}{l}\text { Food, cash Domestic (2), } \\
\text { medicinal }\end{array}$ & A \\
\hline 38 & Phaseolus vulgaris & Fabaceae & Climber & Annual SW & $\begin{array}{l}\text { Food, cash Domestic (2), } \\
\text { medicinal }\end{array}$ & A \\
\hline 39 & Cajanus cajans & Fabaceae & Shrub & $\begin{array}{l}\text { Biannual } \\
\text { SW }\end{array}$ & $\begin{array}{l}\text { Food, cash Domestic (2), } \\
\text { medicinal }\end{array}$ & B \\
\hline 40 & Solanum melanguma & Salanaceae & Shrub & Annual SW & $\begin{array}{l}\text { Food, cash Domestic (2), } \\
\text { medicinal }\end{array}$ & A \\
\hline 41 & Capsicum annиum & Solanaceae & Shrub & Annual W & $\begin{array}{l}\text { Food, cash, domestic } \\
\text { medicinal }\end{array}$ & A \\
\hline 42 & Vernonia amygdalina & Asteraceae & Shrub & Perennial W & $\begin{array}{l}\text { Food, cash, domestic } \\
\text { medicinal }\end{array}$ & A \\
\hline 43 & Ixora coccinea & Rubiaceae & Shrub & Perennial W & Ornamental domestic & B \\
\hline 44 & Aspilia africana & Asteraceae & Shrub & Annual SW & Domestic medicinal & B \\
\hline
\end{tabular}


Table 2: (Continued)

\begin{tabular}{|c|c|c|c|c|c|c|}
\hline 45 & Arachis hypogea & Fabaceae & Shrub & $\begin{array}{l}\text { Biannual } \\
\text { SW }\end{array}$ & $\begin{array}{l}\text { Food, cash, domestic, } \\
\text { industrial }\end{array}$ & $\mathrm{A}$ \\
\hline 46 & Amaranthus species & Amaranthaceae & Forb & Annual $\mathrm{H}$ & Food, domestic & B \\
\hline 47 & Cochorus olitorius & Tiliaceae & Forb & Annual H & Food, domestic & A \\
\hline 48 & Tridax procumbrens & Asteraceae & Forb & Annual $\mathrm{H}$ & Domestic & A \\
\hline 49 & Cucumeropsis manii & Cucurbitaceae & Climber & Annual $\mathrm{H}$ & $\begin{array}{l}\text { Food, cash, domestic, } \\
\text { industrial }\end{array}$ & B \\
\hline 50 & Telfaria occidentalis & Cucurbitaceae & Climber & Annual $\mathrm{H}$ & $\begin{array}{l}\text { Food, cash, domestic, } \\
\text { industrial }\end{array}$ & A \\
\hline 51 & Hibiscus rosasinensis & Malvaceae & Shrub & Perennial W & Ornamental Domestic & B \\
\hline 52 & Ipomoea species & Convolvulaceae & Runner & Annual $\mathrm{H}$ & Domestic & B \\
\hline 53 & Alternanthera species & Amaranthaceae & Shrub & $\begin{array}{l}\text { Perennial } \\
\text { SW }\end{array}$ & Domestic & A \\
\hline 54 & Desmodium scorpiurus & Fabaceae & Climber & Annual H & Domestic & A \\
\hline 55 & Vigna unguiculata & Fabaceae & Shrub & $\begin{array}{l}\text { Biannual } \\
\text { SW }\end{array}$ & Food, cash, Domestic & A \\
\hline 56 & Sida species & Malvaceae & Shrub & Perennial W & Domestic & B \\
\hline 57 & $\begin{array}{l}\text { Talinum triangulare (water } \\
\text { leaf) }\end{array}$ & Portulacaceae & Forb & Annual $\mathrm{H}$ & Food, Domestic & B \\
\hline 58 & Abelmoscus esculentus & Malvaceae & Shrub & $\begin{array}{l}\text { Biannual } \\
\text { SW }\end{array}$ & Food, cash, Domestic & B \\
\hline 59 & Sugar cane & Cane sugar & Grass & Perennial H & Food, industrial cash, & A \\
\hline 60 & Pterocarpus soyauxii & & & & Food, Domestic & B \\
\hline 61 & Alchornea cordifolia & & & & Domestic & A \\
\hline 62 & Napoleona imperialis & & & & Domestic & $\mathrm{C}$ \\
\hline 63 & Terminalia catappa & & & & Food, Domestic & B \\
\hline
\end{tabular}

KEY: A - 70-100\%, B - 40-70\%, C - 10-40\%, D - 0 - 10\%, W - Woody, SW - Semiwoody, H - Herbacerus.

From Table 2 above, with plants from about 30 families and with species per family ranging from $1-10$, for this work alone, the range of plant biodiversity (fodder species) at the disposal of common livestock is large. The implication is that with effective biodiversity conservation, sources of livestock food and by extension the farmer's income and profit is assured. This translates also to a veritable source of protein for the overall good health of the human population. Plant fodder species cut across trees, shrubs, climbers, grasses, forbs et cetera even plants that are termed unwanted. Therefore, biodiversity conservation through reforestation, flooding, erosion control, controlled grazing/wildfires is imperative for sustainability - without which all will be lost. Again, a little over 30 species are perennial - this will ensure year round availability of most fodder species both for the rainy and dry seasons.

Table 3: Potential/possible sources of fodder species for the future.

\begin{tabular}{|c|c|c|c|c|c|c|c|}
\hline $\mathbf{S} / \mathbf{N}$ & $\begin{array}{l}\text { LOCAL } \\
\text { NAME }\end{array}$ & FAMILY & $\begin{array}{l}\text { SCIENTIFIC } \\
\text { NAME }\end{array}$ & $\begin{array}{l}\text { PART } \\
\text { EATEN }\end{array}$ & $\begin{array}{l}\text { GROWTH } \\
\text { FORM }\end{array}$ & CONSUMER & $\begin{array}{l}\text { Other } \\
\text { ECONOMIC } \\
\text { VALUE }\end{array}$ \\
\hline 1 & Ijikere & Anacardaceae & Spondias mombin & $\begin{array}{l}\text { Tender } \\
\text { leaves }\end{array}$ & & $\begin{array}{l}\text { Goat, sheep, } \\
\text { Rabbit }\end{array}$ & Domestic \\
\hline 2 & $\begin{array}{l}\text { Osisi } \\
\text { Akpu }\end{array}$ & Bombacaceae & Ceiba pentandra & $\begin{array}{l}\text { Tender } \\
\text { leaves }\end{array}$ & Tree & $\sqrt{ }$ & Timber \\
\hline 3 & Mbembe & Verbenaceae & Vitex doniana & $\begin{array}{l}\text { Tender } \\
\text { leaves }\end{array}$ & Tree & $\sqrt{ }$ & $\begin{array}{l}\text { Industrial, } \\
\text { Domestic }\end{array}$ \\
\hline 4 & & Malvaceae & $\begin{array}{l}\text { Malvastrum } \\
\text { coromandelianum }\end{array}$ & Fruits & Tree & $\sqrt{ }$ & $\begin{array}{l}\text { Food, cash, } \\
\text { domestic }\end{array}$ \\
\hline 5 & & Poaceae & $\begin{array}{l}\text { Dactyloctenium } \\
\text { aegyptium }\end{array}$ & $\begin{array}{l}\text { Entire } \\
\text { plant }\end{array}$ & Forb & $\sqrt{ }$ & Domestic \\
\hline 6 & & Acanthaceae & Justicia flava & $\begin{array}{l}\text { Entire } \\
\text { plant }\end{array}$ & forb & $\sqrt{ }$ & Domestic \\
\hline 7 & & Verbaceae & $\begin{array}{l}\text { Duranta } \\
\text { repens/erecta }\end{array}$ & Leaf & Shrub & $\sqrt{ }$ & $\begin{array}{l}\text { Ornamental } \\
\text { domestic }\end{array}$ \\
\hline 8 & & Poaceae & $\begin{array}{l}\text { Paspalum } \\
\text { scrobiculatum }\end{array}$ & Meat & & $\sqrt{ }$ & \\
\hline 9 & & Commelinaceae & Commelina diffusa & $\begin{array}{l}\text { Entire } \\
\text { plant }\end{array}$ & Forb & $\sqrt{ }$ & Domestic \\
\hline 10 & & Cyperaceae & Mariscus alternifolias & $\begin{array}{l}\text { Entire } \\
\text { plant }\end{array}$ & Sedge & $\sqrt{ }$ & Domestic \\
\hline 11 & & Cucurbitaceae & & & & $\sqrt{ }$ & \\
\hline 12 & & & Newbouldia laevis & & & $\sqrt{ }$ & Domestic \\
\hline 13 & & & Annona senegalensis & & & $\sqrt{ }$ & Domestic \\
\hline
\end{tabular}


From table 3 above, there are 4 tree, 2 shrubs and 2 forb species mentioned. Goats and sheep in one locality in the

\section{CONCLUSION}

Goats, sheep and Rabbits in the South East feed on a wide variety of fodder species particularly Musa, Zea, Ficus, Grass (Pennisetum, Panicumand Andropogonspp), Persea, Elaeis, Manihot, Colocasia and forbs (Alternanthera, Desmodium, Tridax, Aspilia) et cetera. They are easy to manage, a great source of protein, and an alternative source of income particularly now that crude oil need to be augmented by Agriculture for the Nigerian Economy to become more stable. There are other overlooked sources of fodder spp that need to be revisited: Vitex, Ceiba, Newbouldia, Pterocarpus, Spondias, Terminalia, Moringa, and many other grass and forb species. It is very pertinent to note that most of these fodder species are easily available forest species but presently the forest ecosystem of South East Nigeria is 'threatened' to 'extinction' by powerful negative influence, like serious cattle grazing, incessant forest fire, Erosion and unsustainable Agricultural activities on marginal lands. Saving our forest is synonymous to our survival. south east may savour what is taboo to Goats and sheeps in another locality in the same Southeast.

\section{REFERENCES}

Abbasi, A. M., Khan M.A., Ahmad, M and Zafar, M (2012). Medicinal plant Biodiversity of lesser HimalayasPakistan. Springer Science. 1-4614-1575-6.

Akobundu, I. O. and Agyakwa, C. W. (1998). A handbook of West African Weeds. International Institute of Tropical Agriculture, Ibadan, Nigeria. $565 \mathrm{pp}$.

FAO (1966), "Food and Agriculture Organization of the United Nations" Agricuultural development in Nigeria 1980. Rome, FAO.

FAO (1973). "Food and Agriculture Organization of the United Nations" Production Yearbook, Rome, FAO.

Nyananyo, B. L. (2006). Plants from the Niger Delta. Onyoma Research publications, Port Harcourt, Nigeria. 403pp.

Otegbeye, G. O. and Onyeanusi, A. O. (2006): In: Ivbijaro, M. F. A. Akintola F. and Okechukwu, R. U. E (Eds). Sustainable Environmental Management in Nigeria. Mathivi Production, Ibadan. Nigeria 465 pp.

\section{$\underline{\text { How to cite this article }}$}

Ukpaka, C.G. (2018). Major Sources of Plant Fodder Species of South East Nigeria, Associated Livestock, Percentage Preferences, a Few Physiogenomic Attributes and Potential New Sources. Tropical Journal of Applied Natural Sciences, 2(2): 137-142. Doi: https://doi.org/10.25240/TJANS.2018.2.2.18. 